\title{
High-mass Higgs searches at ATLAS and CMS
}

\author{
Scott Snyder ${ }^{1, a}$, on behalf of the ATLAS and CMS collaborations \\ ${ }^{1}$ PO Box 5000, Upton NY, 11973, USA
}

\begin{abstract}
Several theories beyond the Standard Model, such as the two-Higgs doublet model, predict the existence of high-mass neutral and charged Higgs particles. The ATLAS and CMS experiments have completed an extensive program of searches for such particles using the $\sim 3 \mathrm{fb}^{-1}$ of $\sqrt{s}=13 \mathrm{TeV}$ data recorded during 2015. This note summarizes a selection of these results.
\end{abstract}

\section{Introduction}

Since the first observations of the Higgs boson [1,2], all measurements of its properties have been very consistent with expectations from the Standard Model (SM). There are, however, reasons to suspect that the known electroweak sector of the SM may not be complete, including the unnaturalness of the observed Higgs boson mass and the possibility that new particles could constitute part of the inferred "dark matter" component of the universe. Therefore, both the ATLAS and CMS experiments have been searching for additional heavy Higgs-like scalars. The searches reported here mostly use the $\sim 3 \mathrm{fb}^{-1}$ of $\sqrt{s}=13 \mathrm{TeV}$ data recorded by each experiment during 2015 .

Some searches reported here are interpreted in the context of a simple extension to the SM, the two-Higgs doublet model (2HDM) [3]. In the 2HDM, an additional Higgs doublet is introduced, yielding five physical Higgs bosons: two CP-even scalars $h, H$; one CP-odd scalar $A$; and two charged scalars $H^{ \pm}$. (In the following, the observed $125 \mathrm{GeV}$ resonance is denoted $h ; H$ is a putative heavier resonance.) Besides the particle masses $\left(m_{h}, m_{H}, m_{A}, m_{H^{ \pm}}\right)$, model parameters include the ratio of the vacuum expectation values of the two doublets $\tan \beta$; the mixing angle between $h$ and $H \alpha$; and the potential parameter mixing the two doublets $m_{12}^{2}$. There are many ways in which the doublets can be coupled to other particles. The most studied classes of models are "Type-I," in which all quarks couple to only one doublet, and "Type-II," in which up-type quarks couple to one doublet and downtype quarks to the other. The minimal supersymmetric standard model (MSSM) is a subset of 2HDM, and results are often reported in the context of a set of defined benchmark models $[4,5]$.

\section{Higgs boson decays to $Z Z$}

This section covers decays of a high-mass Higgs boson to two $Z$ bosons, at least one of which decays into a lepton pair. Other diboson decay modes, including $Z \gamma$, are covered in [6].

The $H \rightarrow Z Z \rightarrow 4 \ell$ channel is notable for having a very low background, though the branching ratio is also small. The high-mass analysis for both experiments is based on the analyses for

\footnotetext{
ae-mail: snyder@bnl.gov
} 
$m_{h}=125 \mathrm{GeV}$. Selected events must have two same-flavor, opposite-sign lepton pairs. The CMS analysis [7] requires one pair with $40<m_{\ell \ell}<120 \mathrm{GeV}$ and the other with $12<m_{\ell \ell}<120 \mathrm{GeV}$. Backgrounds are further reduced by requirements on lepton momenta, angular separation, and on track isolation and impact parameter. The resulting background is quite small and consists mostly of $\mathrm{ZZ}$ production, which is estimated from Monte Carlo (MC) simulation. Results are shown in Figure 1, including exclusion limits in $\sigma \times \mathrm{BR}$ for the case of a narrow resonance over the range $200-1000 \mathrm{GeV}$, and also limits for several larger widths as well as an interpretation in terms of a Type-I 2HDM. The corresponding ATLAS analysis is described in [8].
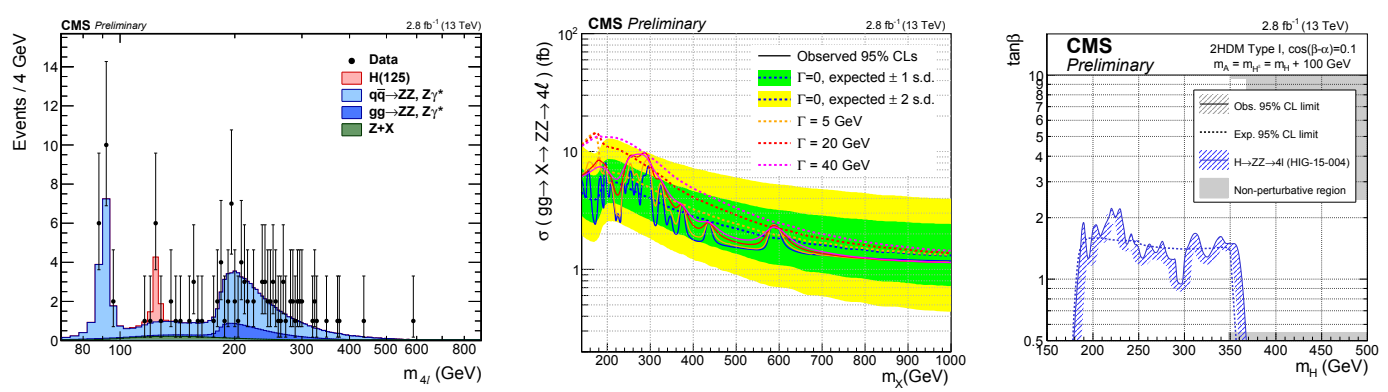

Figure 1. Selected results of the CMS $H \rightarrow Z Z \rightarrow 4 \ell$ search [7]. Left: distribution of four-lepton invariant mass $m_{4 \ell}$. Center: $95 \% \mathrm{CL}$ exclusion in $\sigma \times \mathrm{BR}$ as a function of $m_{4 \ell}$ for the narrow-width approximation and several larger widths. Right: $95 \%$ CL exclusions in $\tan \beta / m_{H}$ for a Type-I $2 \mathrm{HDM}$.

The ATLAS search in the $H \rightarrow Z Z \rightarrow \ell \ell v v$ channel [8] requires a $Z \rightarrow \ell \ell$ decay, $E_{\mathrm{T}}^{\text {miss }}>120 \mathrm{GeV}$ not pointing along a jet, and no $b$-tags. The discriminating variable is $m_{T}^{Z Z}=m_{T}\left(\ell \ell, E_{\mathrm{T}}^{\mathrm{miss}}\right)$. There are additional requirements on $p_{T}^{Z}$, angular separations, and event transverse momentum balance. The major backgrounds are SM diboson production, which is estimated from MC simulations with the $W Z$ background normalized to data, and $Z+$ jets, which is estimated from data. Selected results are shown in Figure 2. The corresponding CMS analysis is described in [9].
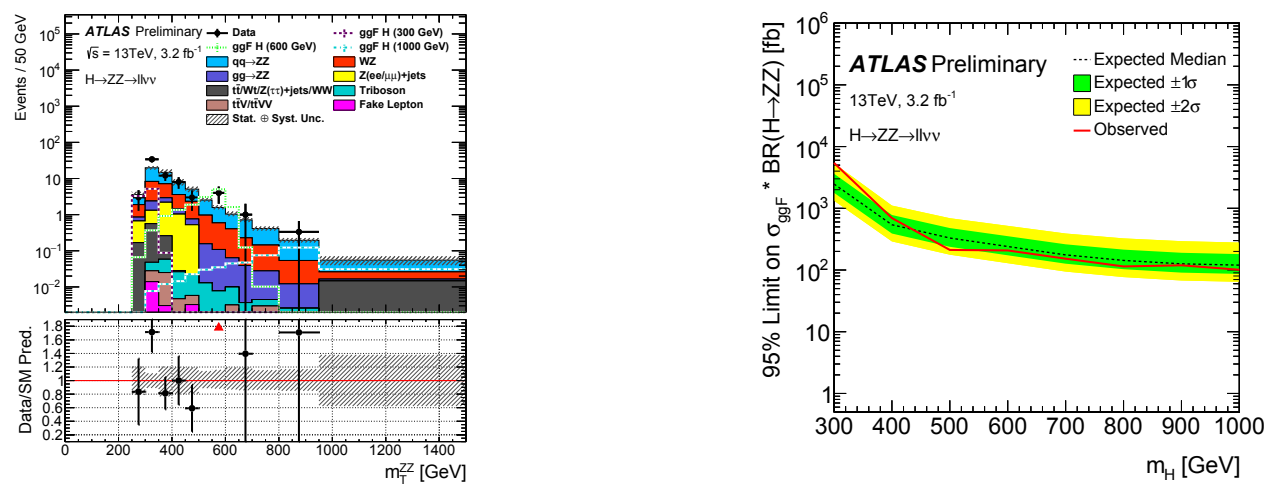

Figure 2. Selected results of the ATLAS $H \rightarrow Z Z \rightarrow \ell \ell v v$ search [8]. Left: $m_{T}^{Z Z}$ distribution. Right: $95 \% \sigma \times \mathrm{BR}$ limit for a narrow-width Higgs boson produced via gluon-gluon fusion. 
In the $H \rightarrow Z Z \rightarrow \ell \ell q q$ channel, both $Z$ bosons are reconstructed; however, there is a large background from $Z+$ jets. For large $m_{H}$, the decay products become boosted, and the jets from the $Z \rightarrow q q$ tend to merge into a single jet. Therefore, the ATLAS $H \rightarrow Z Z \rightarrow \ell \ell q q$ search [10] is divided into "merged" and "resolved" analyses. Besides the $Z \rightarrow \ell \ell$ decay, the merged analysis requires the presence of a large- $R$ jet with $p_{T}>200 \mathrm{GeV}$ that is consistent with originating from $Z$ boson decay, as well as $p_{T}(\ell \ell)>0.3 m_{\ell \ell}$. The resolved analysis requires two jets with $70<m_{j j}<105 \mathrm{GeV}$ and $\sqrt{p_{T}^{2}(\ell \ell)+p_{T}^{2}(j j)}>0.5 m_{\ell \ell j j}$. This analysis is further divided into tagged (with $2 b$-tagged jets) and untagged categories. The dominant backgrounds are $Z+$ jets and $t \bar{t}$, both of which are evaluated from data. Selected results are shown in Figure 3.
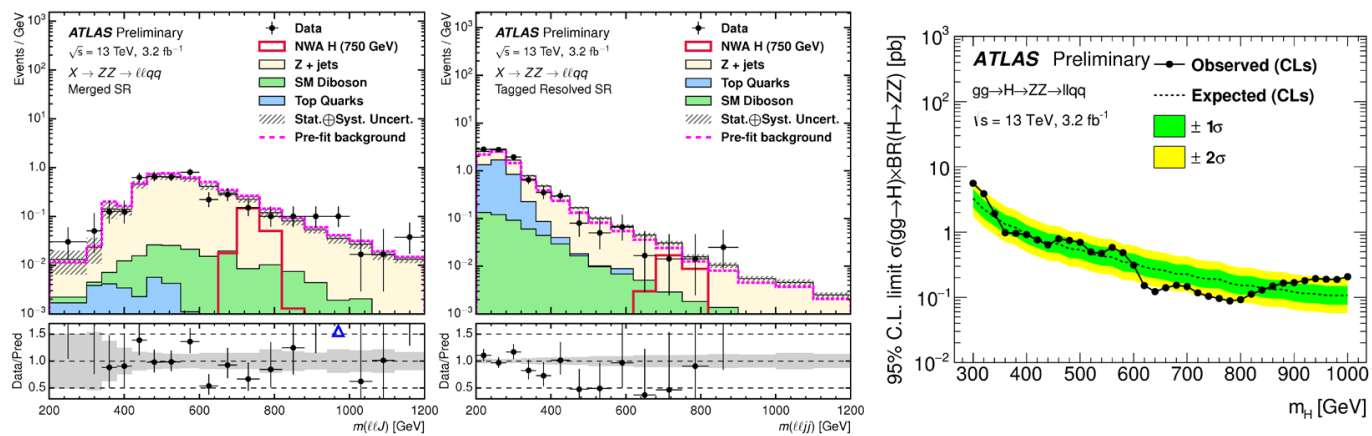

Figure 3. Selected results of the ATLAS $H \rightarrow Z Z \rightarrow \ell \ell q q$ search [10]. Left, middle: Observed $m_{\ell \ell q q}$ distribution compared to background expectations for the (left) merged analysis and (middle) tagged category of the resolved analysis. Right: $95 \%$ CL $\sigma \times$ BR limit for the production of a heavy scalar with narrow width.

\section{Higgs boson decays to other Higgs particles}

This section covers searches involving the CP-odd scalar $A$. Searches for a heavy scalar decaying into two SM Higgs bosons are covered in [11, 12].

CMS has searched for the process $H \rightarrow Z A \rightarrow \ell \ell b b$ [13]. The event selection requires a $Z \rightarrow \ell \ell$ decay (with $75<m_{\ell \ell}<105 \mathrm{GeV}$ ) and two $b$-tagged jets. The analysis proceeds by choosing a set of hypothesized $m_{H} / m_{A}$ pairs. For each specific pair, $\mathcal{H}_{A}^{H}$, a signal region $\mathcal{S}_{A}^{H}$ is defined in the $m_{b b} / m_{\ell \ell b b}$ plane as a rectangle centered on $\mathcal{H}_{A}^{H}$ with sizes given by three times the experimental resolution. The dominant backgrounds are $Z+$ jets and $t \bar{t}$; the background is evaluated by fitting simulated samples to the control region defined by the area of the $m_{b b} / m_{\ell \ell b b}$ plane outside the signal region. (The $m_{\ell \ell}$ invariant mass requirement is also relaxed to $60-120 \mathrm{GeV}$ in the control region.) The $95 \% \mathrm{CL}$ limit on $\sigma \times \mathrm{BR}$ is found for each $\mathcal{H}_{A}^{H}$; selected results are shown in Figure 4 .

ATLAS has also searched for the process $A \rightarrow Z h$, with $h \rightarrow b b$ and either $Z \rightarrow \ell \ell$ or $Z \rightarrow v v$ [14]. Events must have at least one $b$-tagged jet, and are categorized as having either one or two tagged jets. If $p_{T}^{Z}<500 \mathrm{GeV}$ (where $p_{T}^{Z}$ is either $E_{\mathrm{T}}^{\text {miss }}$ or $m_{\ell \ell}$ ), then the analysis requires two jets with

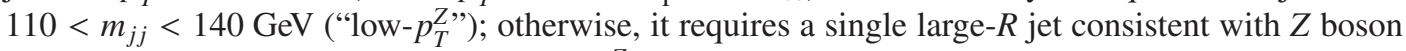

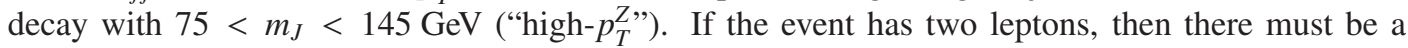
$Z \rightarrow \ell \ell$ decay with $70<m_{\ell \ell}<110 \mathrm{GeV}$ (widened to $55-125 \mathrm{GeV}$ for the high- $p_{T}^{Z}$ case with muons), and in the low- $p_{T}^{Z}$ case $E_{\mathrm{T}}^{\text {miss }} / \sqrt{H_{T}}<3.5 \sqrt{\mathrm{GeV}}$ (to suppress $t \bar{t}$ background). The final discriminant in this case is the overall invariant mass $m(Z h)$. If the event has no leptons, then the analysis requires 

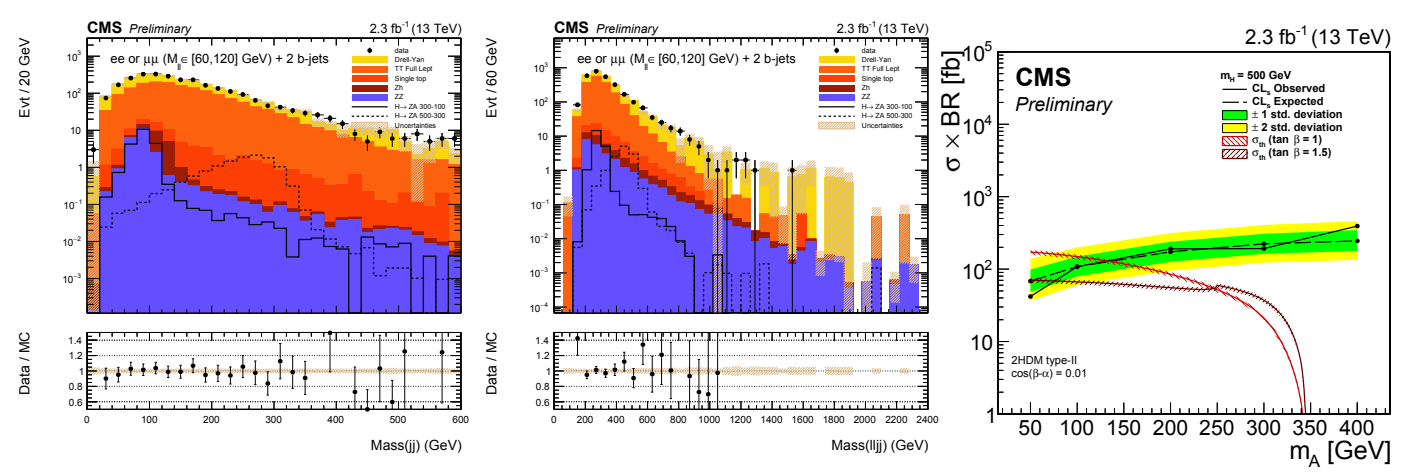

Figure 4. Selected results from the CMS $H \rightarrow Z A \rightarrow \ell \ell b b$ search [13]. Left/middle: $m_{b b} / m_{\ell \ell b b}$ distributions. Right: Resulting $\sigma \times$ BR limit as a function of $m_{A}$ for $m_{H}=500 \mathrm{GeV}$. Expectations for a Type-II 2HDM model are also shown for two different values of $\tan \beta$.

$E_{\mathrm{T}}^{\text {miss }}>150 \mathrm{GeV}$ and, for the low- $p_{T}^{Z}$ category, that the scalar $p_{T}$ sum of the highest three $p_{T}$ jets be $>150 \mathrm{GeV}$ (or $>120 \mathrm{GeV}$ if there are only two jets). Additional requirements are made on the trackbased missing momentum and on the angular separation between objects to reduce backgrounds due to mismeasured $E_{\mathrm{T}}^{\mathrm{miss}}$. The final discriminant for this case is the transverse mass $m_{T}(Z h)$. The dominant backgrounds are $Z+$ jets and $t \bar{t}$ and are studied by defining background-enriched control samples. For the $Z+$ jets background, the $m_{j j}$ (or $m_{J}$ ) requirement is inverted. A two-lepton low- $p_{T}^{Z} t \bar{t}$ control sample is also defined by requiring that the two leptons have different flavor with $m_{e \mu}>40 \mathrm{GeV}$. Backgrounds are estimated from MC simulations, constrained by including the control regions in the final fit. Selected results are shown in Figure 5, including $\sigma \times \mathrm{BR}$ limits as a function of $m_{A}$ and limits in the $\tan \beta / \cos (\beta-\alpha)$ plane in the context of the MSSM.

\section{Higgs boson decays to fermions}

As mentioned earlier, models with two Higgs doublets include charged Higgs bosons. For $m_{H^{ \pm}}>m_{t}$, production is dominantly in association with a top quark, $p p \rightarrow t(b) H^{ \pm}$. Decay is mostly $H^{ \pm} \rightarrow t b$, but $H^{ \pm} \rightarrow \tau v$ is significant for large $\tan \beta$. Both experiments have reported searches for a charged Higgs boson, and also for a heavy $H$ or $A$ scalar decaying to a pair of tau leptons.

CMS has searched for $H^{ \pm} \rightarrow t b$ and $H^{ \pm} \rightarrow \tau v$ produced via $p p \rightarrow t(b) H^{ \pm}$using $8 \mathrm{TeV}$ data from 2012 [15]. The hadronic channel is also sensitive to $t \bar{t} \rightarrow b H^{ \pm} \bar{b} H^{ \pm}$and $t \bar{t} \rightarrow b H^{ \pm} \bar{b} W$. This search is performed in four separate channels.

The first channel is $\tau_{\text {had }}+$ jets, which is sensitive to the $\tau v$ decay mode. This requires a hadronic tau decay, at least three jets, at least one $b$-tagged jet, $E_{\mathrm{T}}^{\mathrm{miss}}>60 \mathrm{GeV}$, and no leptons. Additional requirements are made on the azimuthal separations between objects. The discriminating variable used is $m_{T}\left(\tau_{\text {had }}, E_{\mathrm{T}}^{\text {miss }}\right)$. The dominant backgrounds include SM diboson processes, $W / Z+$ jets, and top quark decays into tau leptons; these are evaluated by replacing the muon in $\mu+$ jets data with a simulated tau lepton. The QCD multijet background is estimated by measuring the rate for a jet to be misidentified as a tau lepton and applying that to multijet data.

The second channel is $\ell \ell$, which is sensitive to both decay modes. This channel requires two opposite-charge leptons not consistent with $Z$ boson decay, at least two jets, $E_{\mathrm{T}}^{\text {miss }}>40 \mathrm{GeV}$, and at least two $b$-tagged jets. The final discriminating variable is the number of $b$-tagged jets. The dominant 

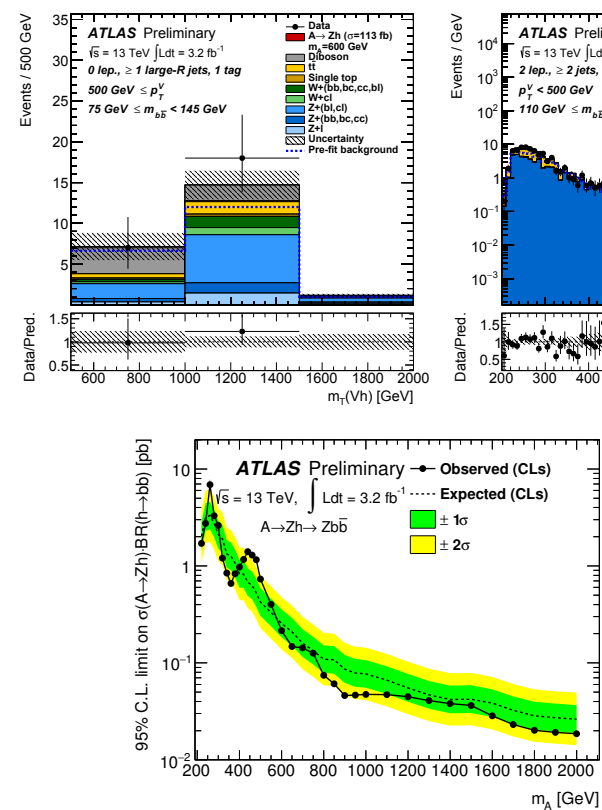
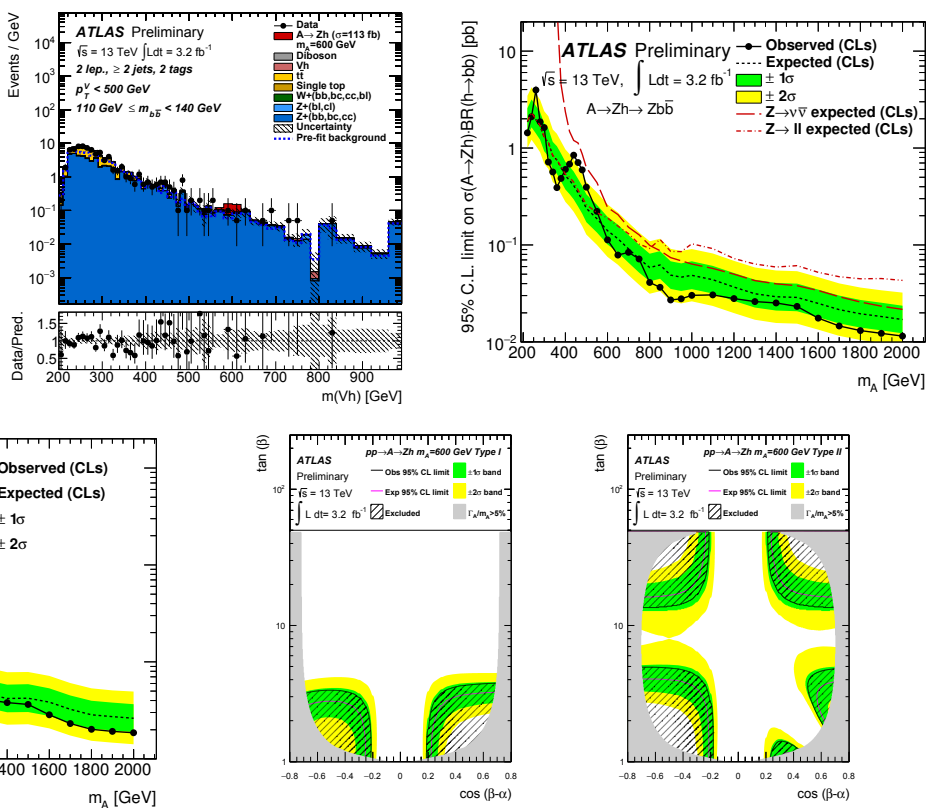

Figure 5. Selected results from ATLAS searches involving a CP-odd scalar $A$ [14]. Top left: $m_{T}(Z h)$ for the 0-lepton, 1-tag, high- $p_{T}^{Z}$ category. Top middle: $m(Z h)$ for the 2-lepton, 2-tag, low- $p_{T}^{Z}$ category. Top right: $95 \%$ $\sigma \times$ BR limit for $A \rightarrow Z h \rightarrow Z b \bar{b}$ as a function of $m_{A}$, for production via the gluon-gluon fusion process. Bottom left: Same, for production with associated $b$ quarks. Bottom middle: Interpretation of the $A \rightarrow Z h \rightarrow Z b \bar{b}$ search in terms of a Type-I 2HDM. Bottom right: Same for Type-II.

background is $t \bar{t}$ decay with two leptons; backgrounds are evaluated from MC simulations. The third channel is the same except that instead of two leptons, a muon and a tau lepton are required, and only one $b$-tagged jet is required. Backgrounds are again evaluated from MC, convoluted with the probability for a jet to be misidentified as a tau lepton, which is measured from data.

The fourth channel is $\ell+$ jets, which is sensitive to the $t b$ decay mode. Events must have one lepton, $E_{\mathrm{T}}^{\text {miss }}>20 \mathrm{GeV}$, at least two jets, and at least one $b$-tagged jet. Events are categorized based on whether they have one or two $b$-tagged jets. Background shapes are taken from MC simulations and normalized by fitting to data in both the signal and several control regions.

Selected results from this search are shown in Figure 6.

ATLAS has also searched for $H^{ \pm} \rightarrow \tau v$ using $13 \mathrm{TeV}$ data [16]. (The ATLAS $H^{ \pm} \rightarrow t b$ search using $8 \mathrm{TeV}$ data [17] is not covered here.) To select the final state $(t \rightarrow j j b) b \tau_{\text {had }} v$, events must contain a hadronically-decaying tau lepton, no other leptons, at least three jets with at least one $b$ tagged jet, and $E_{\mathrm{T}}^{\text {miss }}>150 \mathrm{GeV}$. The final discriminant is the transverse mass $m_{T}\left(\tau_{\text {had }}, E_{\mathrm{T}}^{\text {miss }}\right)$, which must be $>50 \mathrm{GeV}$. The dominant backgrounds are those with a real $\tau_{\text {had }}$, including $W \rightarrow \tau v$ and $t \bar{t}$; these are estimated with MC simulation and validated with data. Backgrounds with jets misidentified as tau leptons are estimated using data, while backgrounds with other leptons misidentified as tau leptons are small and are also estimated with MC simulation. Selected results are shown in Figure 7.

Both CMS and ATLAS have also searched for a heavy scalar decaying into two tau leptons. This is particularly interesting in the context of the MSSM, where the couplings of Higgs bosons 

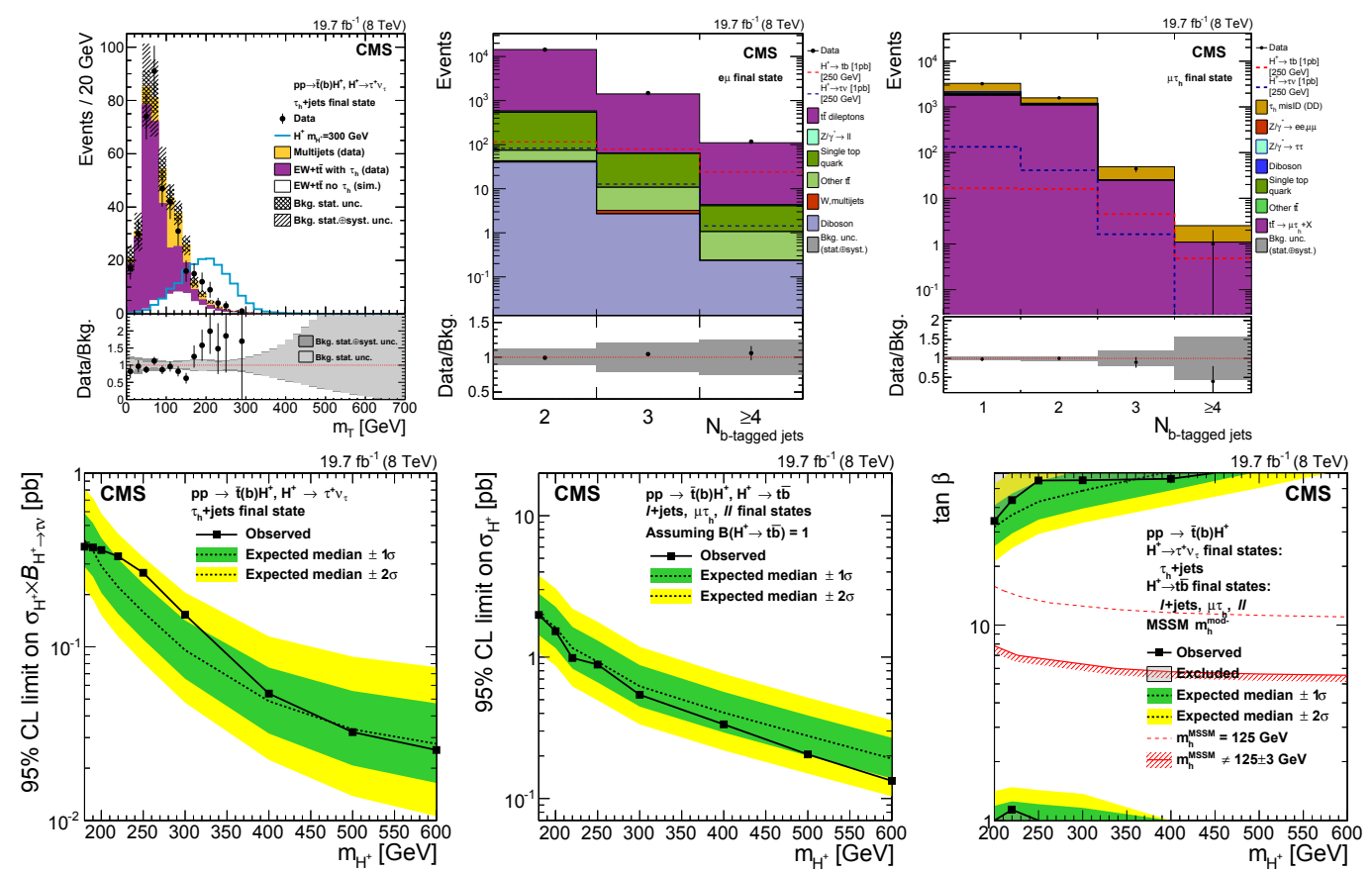

Figure 6. Selected results from the CMS charged Higgs boson search [15]. Top: observed data compared to MC simulations in the (left) $\tau_{\text {had }}+$ jets, (middle) $e \mu$, and (right) $\mu \tau_{\text {had }}$ channels. Bottom: $95 \% \sigma \times$ BR limit as a function of $m_{H^{ \pm}}$for the (left) $\tau_{\text {had }}+$ jets channel and (middle) the other three channels. Bottom right: $95 \% \mathrm{CL}$ exclusion in $\tan \beta / m_{H^{ \pm}}$for the MSSM benchmark model $m_{h}^{\bmod ^{-}}$.

to tau leptons may be enhanced for large $\tan \beta$. The CMS search [18] is divided into four channels: $\tau_{e} \tau_{\text {had }}, \tau_{\mu} \tau_{\text {had }}, \tau_{e} \tau_{\mu}$, and $\tau_{\text {had }} \tau_{\text {had }}$. The two tau lepton candidates must have opposite sign and be wellseparated in $\Delta R$ ( $>0.3$ for $\tau_{e} \tau_{\mu}$ and $>0.5$ for other channels). In the $\tau_{e} \tau_{\text {had }}$ and $\tau_{\mu} \tau_{\text {had }}$ channels, there must be no opposite-sign $e e$ or $\mu \mu$ pairs and also $m_{T}\left(\ell, E_{\mathrm{T}}^{\mathrm{miss}}\right)<40 \mathrm{GeV}(e)$ or $<30 \mathrm{GeV}(\mu)$. An additional topological requirement is applied in the $\tau_{e} \tau_{\mu}$ channel to reject $t \bar{t}$ events, and events are then categorized as having either 0 or $\geq 1 b$-tagged jets. The final discriminant is the $\tau \tau$ transverse mass $m_{T, \tau \tau}$, computed using the likelihood technique described in [19]. The dominant $Z \rightarrow \tau \tau$ background is evaluated using MC simulations and cross-checked with $Z \rightarrow \mu \mu$ data. Other backgrounds include $t \bar{t}$, also evaluated using MC and checked against data, and $W+$ jets and multijets, which are evaluated using same-sign $\tau_{\text {had }}$ candidates and high $-m_{T}$ control regions. Selected results are shown in Figure 8.

The ATLAS $H / A \rightarrow \tau \tau$ search [20] is divided into two channels. The first requires exactly one electron or muon along with a $\tau_{\text {had }}$ candidate. Additional requirements include $\Delta \phi\left(\tau_{\text {had }}, \ell\right)>2.4$, $m_{T}\left(\ell, E_{\mathrm{T}}^{\mathrm{miss}}\right)<40 \mathrm{GeV}$, and for electrons, $m\left(e, \tau_{\text {had }}\right)$ must be outside the range $80-110 \mathrm{GeV}$, to reduce background from $Z \rightarrow e e$. The second channel requires two $\tau_{\text {had }}$ candidates with opposite charge, leading/subleading $p_{T}>110 / 55 \mathrm{GeV}$, and $\Delta \phi\left(\tau_{\text {had }}, \tau_{\text {had }}\right)>2.7$. For both channels, events are categorized as either $b$-veto (no $b$-tagged jets) or $b$-tagged (at least one $b$-tagged jet). The final discriminant is $\left(m_{T}^{\text {tot }}\right)^{2}=m_{T}^{2}\left(E_{\mathrm{T}}^{\text {miss }}, \tau_{1}\right)+m_{T}^{2}\left(E_{\mathrm{T}}^{\text {miss }}, \tau_{2}\right)+m_{T}^{2}\left(\tau_{1}, \tau_{2}\right)$. The dominant background for the $\tau_{\ell} \tau_{\text {had }}$ channel is $Z \rightarrow \tau \tau$, while that for the $\tau_{\text {had }} \tau_{\text {had }}$ channel is multijets. Backgrounds containing real $\tau_{\text {had }}$ are estimated from MC simulations. Those with misidentified $\tau_{\text {had }}$ are estimated using a "fake-factor" method 

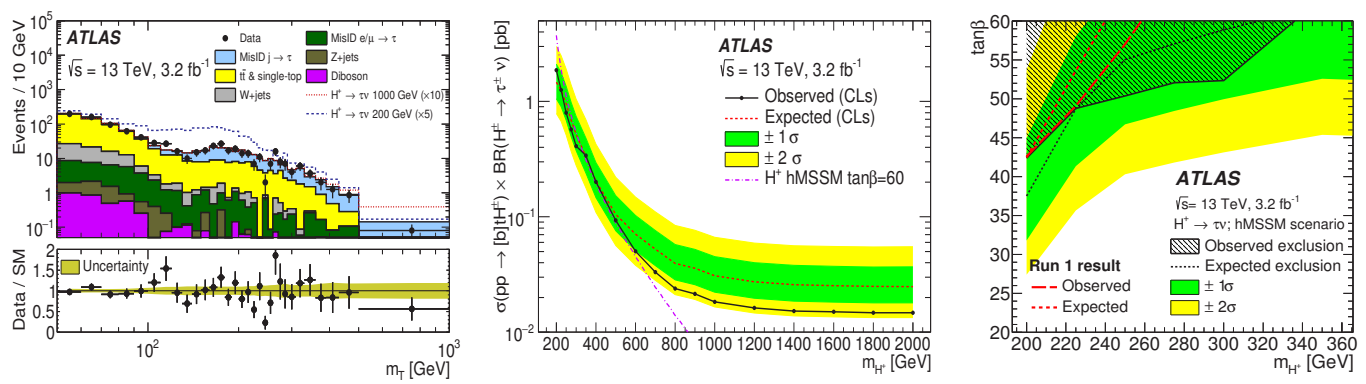

Figure 7. Selected results from the ATLAS charged Higgs boson search [16]. Left: $m_{T}$ distribution after event selection. Middle: 95\% $\sigma \times$ BR limit for charged Higgs boson production. Right: 95\% exclusion limits in $\tan \beta / m_{H^{ \pm}}$for the hMSSM scenario.
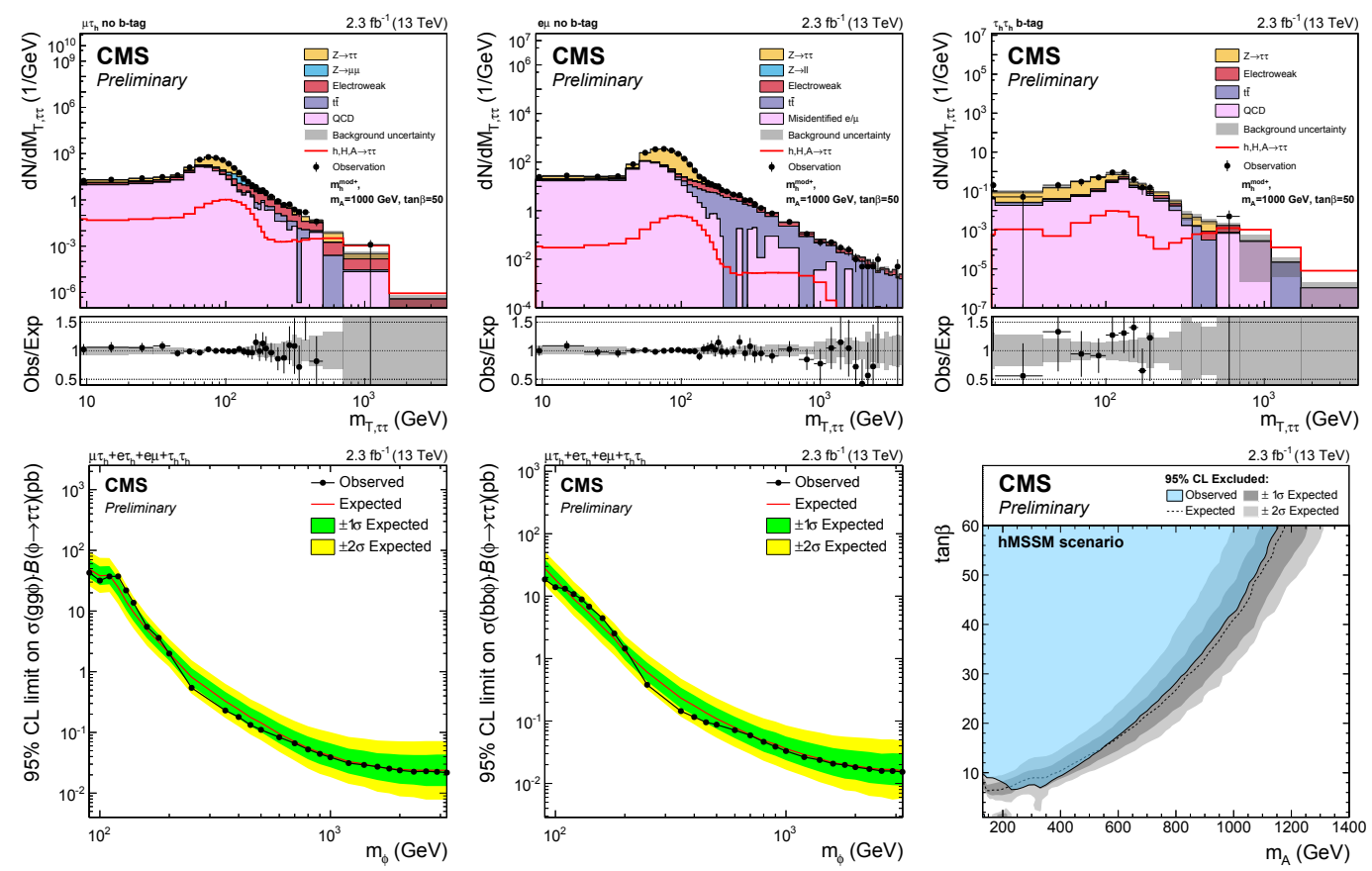

Figure 8. Selected results from the CMS $H / A \rightarrow \tau \tau$ search [18]. Top: Distributions of $m_{T, \tau \tau}$ for data and expected background for the (left) untagged $\tau_{\mu} \tau_{\text {had }}$, (middle) untagged $\tau_{e}, \tau_{\mu}$, and (right) tagged $\tau_{\text {had }} \tau_{\text {had }}$ channels. Bottom left, middle: $95 \% \sigma \times$ BR limits as a function of $m_{\phi}$ (where $\phi$ denotes either $H$ or $A$ ) for (left) production via gluon-gluon fusion and (middle) associated production with $b$ quarks. Bottom right: $95 \%$ exclusions in $\tan \beta / m_{A}$ for the hMSSM scenario.

in which a control sample, not selected for tau leptons, is convoluted with the measured probability for a jet to be misidentified as a tau lepton. Selected results are shown in Figure 9. 

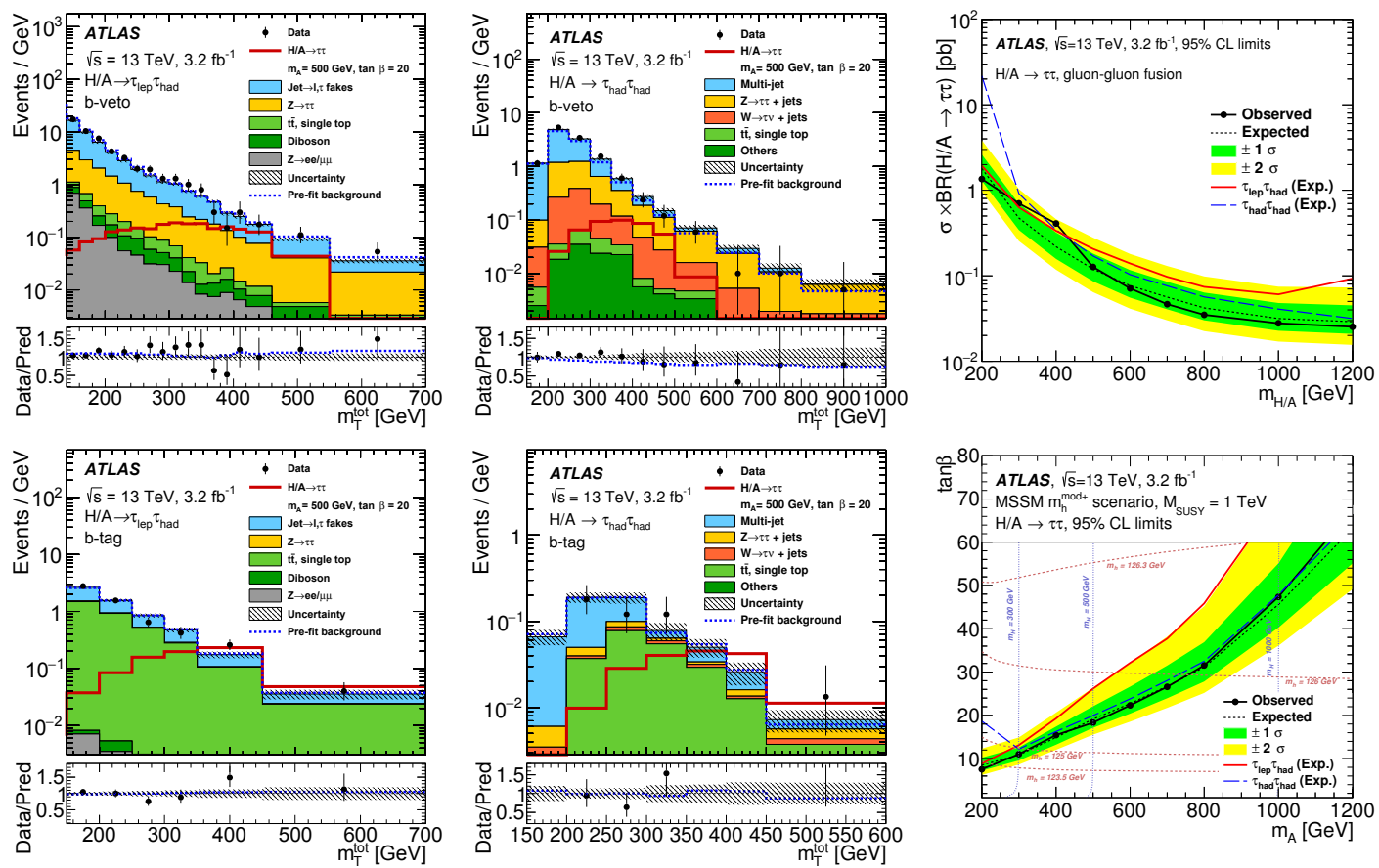

Figure 9. Selected results from the ATLAS $H / A \rightarrow \tau \tau$ search [20]. Left, middle: Distributions of $m_{T}^{\text {tot }}$ for the (left) $\tau_{\ell} \tau_{\text {had }}$ and (right) $\tau_{\text {had }} \tau_{\text {had }}$ channels for the (top) $b$-veto and (bottom) $b$-tag categories. Top right: $95 \% \sigma \times \mathrm{BR}$ limit for $H / A \rightarrow \tau \tau$ as a function of the scalar mass, assuming production via gluon-gluon fusion. Bottom right: 95\% limits in $\tan \beta / m_{A}$ for the MSSM $m_{h}^{\bmod +}$ scenario.

\section{Higgs boson decays to invisible particles}

There is good evidence from astrophysics that so-called "dark matter" makes up a substantial fraction of the universe. If some portion of this is composed of a new, massive particle, then it should couple with the Higgs sector and, depending on its mass, may have a significant decay branching ratio. In fact, some models such as supersymmetry [21] and large extra dimensions [22, 23], naturally include heavy scalars coupling to invisible particles.

CMS has searched for a heavy scalar decaying to undetected particles, in both cases assuming that the heavy scalar decays exclusively to such particles. Two strategies are used to tag such decays. In the first, CMS searches for an invisibly-decaying scalar produced in association with a $Z$ boson that decays into two leptons [24]. This search requires a $Z \rightarrow \ell \ell$ decay with $m_{\ell \ell}$ within $15 \mathrm{GeV}$ of the nominal $Z$ boson mass, $E_{\mathrm{T}}^{\text {miss }}>100 \mathrm{GeV}$, no more than one jet with $p_{T}>30 \mathrm{GeV}$, and no $b$-tagged jets or additional leptons. Additional requirements are $\Delta \phi\left(\ell \ell, E_{\mathrm{T}}^{\mathrm{miss}}\right)>2.8$ and $\left|E_{\mathrm{T}}^{\mathrm{miss}}-p_{T}^{\ell \ell}\right| / p_{T}^{\ell \ell}<0.4$. Events are split into zero- and one-jet categories. The final discriminating variable is $m_{T}\left(\ell \ell, E_{\mathrm{T}}^{\mathrm{miss}}\right)$, which is required to be above $200 \mathrm{GeV}$. The dominant background is $Z+$ jets, which is estimated using a control sample of $\gamma+$ jets events. Other backgrounds are estimated from a control sample in which the two leptons are different flavors. Results are shown in Figure 10; $\sigma \times$ BR limits are given for the heavy scalar mass range $125-600 \mathrm{GeV}$. 
The second CMS search is for an invisibly-decaying heavy scalar produced via vector boson fusion (VBF) [25]. This search requires the presence of a VBF jet pair consisting of two forward jets with $\Delta \eta_{j j}>3.6$ and $m_{j j}>1100 \mathrm{GeV}$. In addition, there must be no leptons, $E_{\mathrm{T}}^{\text {miss }}>200 \mathrm{GeV}$, and $\Delta \phi\left(j, E_{\mathrm{T}}^{\text {miss }}\right)>2.3$. The dominant $W / Z+$ jets background is evaluated using four control regions. One of these requires the presence of a $Z \rightarrow \mu \mu$ decay, and the other three require the presence of a single electron, muon, or tau lepton, respectively. The QCD multijet background is addressed with a fifth control region defined by inverting the $\Delta \phi$ requirement. The data are fit to simulated backgrounds in all five control regions simultaneously along with the signal region and the result found by counting the number of data and fit background events in the signal region. Results are shown in Figure 10.
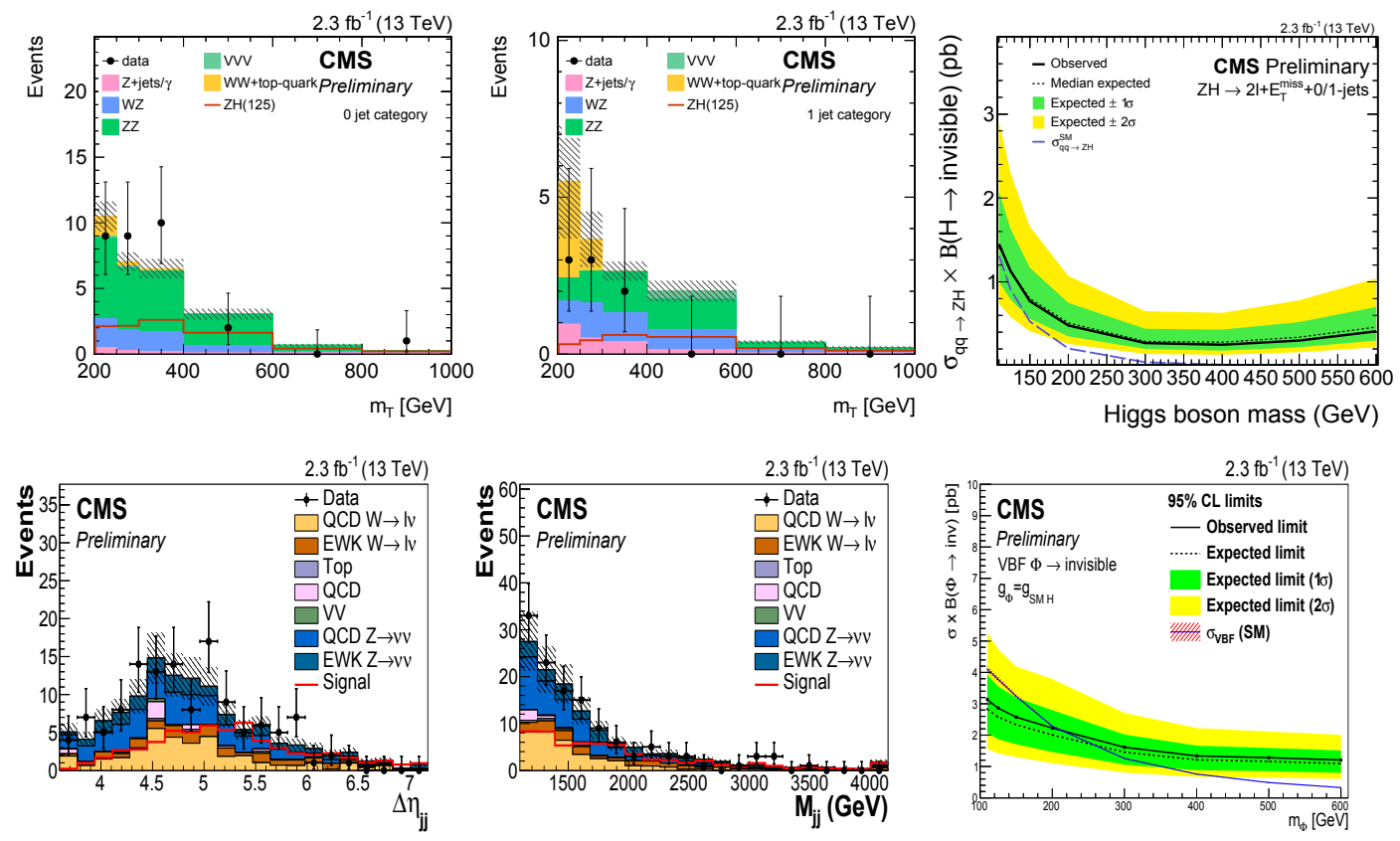

Figure 10. Selected results from the CMS searches for a heavy scalar decaying to invisible particles. Top row: Associated production with $Z \rightarrow \ell \ell$ [24]. Left/middle: $m_{T}\left(\ell \ell, E_{\mathrm{T}}^{\mathrm{miss}}\right)$ for the 0 - and 1-jet categories. Right: 95\% $\sigma \times$ BR limits. Bottom row: Production via vector boson fusion [25]. Left/middle: Distributions of $\Delta \eta_{j j}$ and $m_{j j}$ for the VBF pair. Right: $95 \% \sigma \times$ BR limits.

\section{Summary}

ATLAS and CMS have completed an extensive set of searches for new heavy scalars using the $3 \mathrm{fb}^{-1}$ of $\sqrt{s}=13 \mathrm{TeV}$ data recorded in 2015. No significant excesses above SM expectations have been found so far. However, the LHC continues to perform exceptionally well, and the searches will continue with future data.

This work is supported in part by the U.S. Department of Energy under contract DE-AC0298CH10886 with Brookhaven National Laboratory. 


\section{References}

[1] ATLAS Collaboration, Phys. Lett. B 716, 1 (2012), 1207.7214

[2] CMS Collaboration, Phys. Lett. B 716, 30 (2012), 1207.7235

[3] G. Branco et al., Phys. Rep. 516, 1 (2012), 1106.0034

[4] M. Carena et al., Eur. Phys. J. C73, 2552 (2013), 1302 . 7033

[5] A. Djouadi et al., Eur. Phys. J. C73, 2650 (2013), 1307.5205

[6] G. Halladjian, Search for new resonances with masses between $500 \mathrm{GeV}$ and $1 \mathrm{TeV}$ with final states other than two photons (ATLAS+CMS), in these Proceedings

[7] CMS Collaboration, Studies of Higgs boson production in the four-lepton final state at $\sqrt{s}=$ $13 \mathrm{TeV}$, CMS-PAS-HIG-15-004 (2015), http://cdsweb.cern.ch/record/2139978

[8] ATLAS Collaboration, Search for high-mass resonances decaying into a $Z$ boson pair in the $\ell \ell v v$ final state in pp collisions at $\sqrt{s}=13 \mathrm{TeV}$ with the ATLAS detector, ATLAS-CONF-2016-012 (2016), http: //cdsweb.cern.ch/record/2140833

[9] CMS Collaboration, Search for a heavy scalar boson decaying into a pair of $Z$ bosons in the $2 \ell 2 v$ final state, CMS-PAS-HIG-16-001 (2016), http: //cdsweb. cern. ch/record/2140099

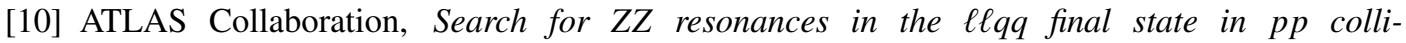
sions at $\sqrt{s}=13 \mathrm{TeV}$ with the ATLAS detector, ATLAS-CONF-2016-016 (2016), http://cdsweb.cern.ch/record/2141005

[11] X. Sun, Search for HH production by the ATLAS collaboration, in these Proceedings

[12] A. Pozdnyakov, Search for HH production by the CMS collaboration, in these Proceedings

[13] CMS Collaboration, Search for $p p \rightarrow H \rightarrow l^{+} l^{-} b \bar{b}$ with 2015 data, CMS-PAS-HIG-16-010 (2016), http://cdsweb.cern.ch/record/2140613

[14] ATLAS Collaboration, Search for a CP-odd Higgs boson decaying to Zh in pp collisions at $\sqrt{s}=13$ TeV with the ATLAS detector, ATLAS-CONF-2016-015 (2016), http://cdsweb. cern.ch/record/2141003

[15] CMS Collaboration, JHEP 11, 018 (2015), 1508.07774

[16] ATLAS Collaboration, Phys. Lett. B 759, 555 (2016), 1603.09203

[17] ATLAS Collaboration, JHEP 03, 127 (2016), 1512 . 03704

[18] CMS Collaboration, Search for a neutral MSSM Higgs boson decaying into $\tau \tau$ at $13 \mathrm{TeV}$, CMSPAS-HIG-16-006 (2016), http: //cdsweb. cern. ch/record/2160252

[19] CMS Collaboration, JHEP 10, 160 (2014), 1408. 3316

[20] ATLAS Collaboration (2016), 1608.000890

[21] G. Belanger et al., Phys. Lett. B519, 93 (2001), hep-ph/0106275

[22] G.F. Giudice et al., Nucl. Phys. B595, 250 (2001), hep-ph/0002178

[23] M. Battaglia et al., The Invisible Higgs decay width in the add model at the LHC, in Physics at TeV colliders. Proceedings, Workshop, Les Houches, France, May 26-June 3, 2003 (2004), hep-ph/0402062

[24] CMS Collaboration, Search for invisible Higgs bosons in $p p \rightarrow Z H \rightarrow 2 \ell+E_{\mathrm{T}}^{\text {miss }}$ channels at $\sqrt{s}=13 \mathrm{TeV}$, CMS-PAS-HIG-16-008 (2016), http://cdsweb. cern. ch/record/2138371

[25] CMS Collaboration, Search for invisible decays of a Higgs boson produced via vector boson fusion at $\sqrt{s}=13 \mathrm{TeV}$, CMS-PAS-HIG-16-009 (2016), http://cdsweb. cern.ch/record/2142460 